\title{
IdeAs
}

Idées d'Amériques

18 | 2021

Frontières dans les Amériques - Intégration, sécurité et migrations

\section{Introduction. Amérique centrale, 1821-2021 : un bicentenaire en ordre dispersé}

Félix Chirú Barrios et David Marcilhacy

\section{(2) OpenEdition}

\section{Journals}

Édition électronique

URL : https://journals.openedition.org/ideas/11453

DOI : 10.4000/ideas. 11453

ISSN : 1950-5701

Éditeur

Institut des Amériques

Référence électronique

Félix Chirú Barrios et David Marcilhacy, « Introduction. Amérique centrale, 1821-2021 : un bicentenaire en ordre dispersé », IdeAs [En ligne], 18 | 2021, mis en ligne le 01 octobre 2021, consulté le 21 octobre 2021. URL : http://journals.openedition.org/ideas/11453; DOI : https://doi.org/10.4000/ideas.11453

Ce document a été généré automatiquement le 21 octobre 2021.

\section{c) (†)}

IdeAs - Idées d'Amériques est mis à disposition selon les termes de la licence Creative Commons Attribution - Pas d'Utilisation Commerciale - Pas de Modification 4.0 International. 


\title{
Introduction. Amérique centrale, 1821-2021 : un bicentenaire en ordre dispersé
}

\author{
Félix Chirú Barrios et David Marcilhacy
}

1 Invisibilisée ou méconnue, l'Amérique centrale échappe trop souvent aux radars des médias ou n'apparaît - y compris dans la production scientifique - qu'au travers de crises ou de phénomènes endémiques tels que la violence, la mauvaise gouvernance, le drame migratoire ou les catastrophes climatiques. Le Bicentenaire de l'Indépendance, célébré à travers toute la région en cette année 2021 et dont le pic a eu lieu le 15 septembre ${ }^{1}$, constitue de ce point de vue un moment paradoxal: temps fort dans l'histoire des nations centraméricaines, sa commémoration est un moment privilégié de construction des récits nationaux - donnant à voir le chemin parcouru au cours de ces 200 ans d'indépendance vis-à-vis de l'Espagne - et offre, dans le même temps, à travers ses modalités festives et les circonstances qui l'accompagnent, un condensé des maux dont souffre la région.

2 Ce dossier porte un regard régional sur les commémorations du Bicentenaire qui ont jalonné l'année 2021. Précisément parce que cet anniversaire se situe aujourd'hui dans un contexte de déplacement des enjeux à une échelle globale, la perspective ici adoptée propose une vision large de la région centraméricaine, étendue à la fois au Chiapas qui faisait partie de l'ancienne Capitainerie générale du Guatemala jusqu'en 1821, avant son annexion à la fédération du Mexique en 1824 - et au Panama - pays situé géographiquement en Amérique centrale mais dont la trajectoire historique l'a durablement séparé des autres territoires de l'Isthme.

Comme il ressort des articles ici réunis, les commémorations de l'Indépendance ont été dominées par la pandémie de Covid-19 et ses effets dans la région. Passé à un second plan face à la crise sanitaire, le Bicentenaire n'a pas donné lieu à des festivités réunissant toute la région autour de ses deux siècles d'indépendance. Il a été placé sous le signe de commémorations éparses, marquées par une forme d'improvisation et dominées par les incertitudes du futur. Parmi elles, l'impact économique de la 
pandémie, les crises politiques que traverse la région ou les frustrations causées par les problèmes structurels dont souffrent ces pays, à commencer par les inégalités sociales, un phénomène ancien mais croissant.

4 S'il est vrai que le monde académique a offert un espace de discussion et de réflexion autour de ces 200 ans d'indépendance - le plus souvent en modalité virtuelle -, les gouvernements n'ont en revanche pas accordé à cette commémoration toute son importance historique, renonçant à se saisir de cette opportunité pour confronter les racines historiques des questions auxquelles est confrontée la région et dessiner des perspectives d'avenir. Une opportunité qu'ont néanmoins saisie certains collectifs pour mettre en avant leurs revendications, qu'il s'agisse des droits et de l'identité des peuples autochtones face aux phénomènes d'exclusion sociale et de racisme systémique, comme ce fut le cas au Guatemala, ou pour dénoncer la corruption endémique et la dérive autoritaire, comme l'ont illustré les protestations au Salvador. En ce sens, on a assisté en 2021 à une commémoration en ordre dispersé, limitée non seulement par la pandémie mais aussi par un climat d'insatisfaction, voire de désespoir, lié au manque de perspectives d'avenir face aux faiblesses démocratiques et à l'absence d'un horizon meilleur. Le Panama, dont l'essentiel des célébrations a été repoussé au 28 novembre ${ }^{2}$, n'a-t-il pas suspendu ses traditionnels défilés civiques ? Signe des temps, le gouvernement panaméen a néanmoins ouvert une plateforme digitale baptisée Ágora, destinée à recueillir les initiatives citoyennes pour résoudre les injustices et dettes sociales héritées de l'histoire de l'Isthme, dans le cadre d'un programme au nom éloquent : Pacto del Bicentenario. Cerrando brechas.

5 Pour ouvrir ce dossier, l'historien David Díaz Arias offre une analyse transversale des commémorations qui ont eu lieu dans la région, marquées par l'impact de la pandémie, la crise économique et le retard des gouvernements à nommer les commissions chargées d'organiser ces célébrations. Il souligne combien les modalités commémoratives et les discours ont été dictés par les intérêts nationaux et immédiats de chacun des pays, au détriment d'un programme commun qui aurait débattu du passé et suscité une réflexion collective sur les défis pour le futur de la région. Un bilan qu'il conclut en interrogeant la viabilité des États-nations de l'Amérique centrale - une région divisée au sens "symbolique et social» - dans un monde complexe et interdépendant.

6 Clara Duterme aborde, quant à elle, le Bicentenaire au Guatemala en soulignant la contradiction entre les velléités du gouvernement d'en faire une célébration de l'unité nationale et la réalité du climat social, marqué par des inégalités croissantes, les insuffisances des politiques publiques pour promouvoir la réconciliation après les accords de paix des années 1990 et un évident manque de confiance dans les institutions. Elle souligne ainsi le décalage entre le discours officiel et les revendications de larges franges de la population, que peut résumer le slogan « 200 ans de racisme, de persécution, de génocide, de corruption, de pillage et d'impunité ».

7 Connu pour constituer une exception dans la région centraméricaine, eu égard à sa longue tradition démocratique et à la relative stabilité de ses institutions, le Costa Rica a commémoré le Bicentenaire là encore sous le signe de la pandémie et dans un climat d'apathie face à l'importance historique de cette date. C'est ce qui ressort de l'analyse proposée par Eduardo Madrigal, qui souligne l'influence d'un contexte national éprouvé par l'impact des réformes néolibérales et les accords de libre échange. Malgré certaines initiatives intéressantes émanant du monde universitaire, les déclarations 
officielles s'en sont tenues à un discours convenu destiné à mettre en valeur les politiques publiques pratiquées par les gouvernements successifs en matière de développement durable et de promotion d'un Costa Rica créatif et pluriel, illustrant par là-même l'absence de réflexion construite sur l'Indépendance et l'épuisement du récit national.

Dans leur analyse de la commémoration du Bicentenaire au Chiapas, Amanda Úrsula Torres Freyermuth et Armando Méndez Zárate mettent en lumière les liens distendus que ce territoire entretient avec son passé historique qui l'unit à la Capitainerie générale du Guatemala, préférant souligner l'importance de son rattachement au Mexique depuis 1824. Le Chiapas peut pourtant être considéré comme le pionnier du mouvement émancipateur centraméricain, ayant proclamé son indépendance dès le 28 août 1821 dans la ville de Comitán. Néanmoins, le Bicentenaire laisse un goût d'inabouti à ceux qui, depuis le monde académique ou certains collectifs citoyens, regrettent le désintérêt dont ont fait preuve les autorités face aux enjeux de cette commémoration. Un débat qui peut-être ressurgira en 2024 lors des probables célébrations à venir du bicentenaire de l'annexion du Chiapas au Mexique.

En définitive, on pourrait penser que les commémorations du Bicentenaire de l'Indépendance auraient pu constituer un moment privilégié pour repenser le passé de la région et proposer un projet commun et inclusif face aux défis auxquels ces pays sont confrontés aujourd'hui. Mais n'est-ce pas le propre de toute célébration mémorielle que de manquer son objectif, qu'il s'agisse de renforcer la cohésion nationale ou d'exalter le récit patriotique ? En cette année 2021, la pandémie a partiellement gommé ce que sont d'habitude les guerres de mémoire associées à toute commémoration. Ne peut-on parler alors d'occasion manquée, tandis que les célébrations organisées jusqu'à ce jour ont plutôt été dominées par une forme de désillusion, aussi bien exprimée par le manque d'enthousiasme institutionnel que par la relative indifférence des sociétés concernées?

\section{NOTES}

1. La date du 15 septembre est symbolique des débuts du processus d'indépendance de la Nouvelle Espagne, dont faisaient partie les territoires centraméricains à travers la Capitainerie générale du Guatemala. Par ailleurs, le 15 septembre 1821, la Députation provinciale du Guatemala signa son Acte d'Indépendance vis-à-vis de la Monarchie espagnole, invitant les autres provinces de la Capitainerie à la rejoindre.

2. Le 28 novembre 1821 est la date anniversaire de l'indépendance du Commandement général de Tierra Firme, juridiction correspondant à l'ancienne Audience royale de Panama et placée au XVIII ${ }^{\mathrm{e}}$ siècle sous la dépendance de la Vice-Royauté de Nouvelle Grenade. 


\section{AUTEURS}

\section{FÉLIX CHIRÚ BARRIOS}

Professeur titulaire à l'Université de Panama, Félix Chirú Barrios a obtenu un doctorat en Histoire à l'Université du Costa Rica. Ancien boursier de l'Office allemand d'échanges universitaires (DAAD), il a été effectuer un séjour à l'Institut ibéro-américain de Berlin et a géré la Chaire centraméricaine entre 2010 et 2015. Il publie régulièrement des articles historiques pour le journal La Estrella de Panamá. Paru dans l'ouvrage La justa libertad de los pueblos (Madrid, 2021), son dernier article a pour titre : « Entre La Epopeya del Istmo a Yo defiendo mi patria. Centenario y Sesquicentenario de la Independencia de Panamá ». chirufelix4@hotmail.com

\section{DAVID MARCILHACY}

David Marcilhacy est Professeur des universités en Histoire et civilisation de l'Espagne et des mondes ibériques contemporains à Sorbonne Université. Directeur adjoint de l'équipe du CRIMIC, il consacre ses recherches, dans une perspective d'histoire globale, aux imaginaires (trans)nationaux à l'œuvre en Espagne, au Panama et dans les sociétés hispanophones contemporaines (hispanisme, ibérisme, panaméricanisme), ainsi qu'à la dimension culturelle des relations internationales (politiques symboliques, politiques de mémoire, diplomatie culturelle, géopolitique). Il est l'auteur de différentes publications, dont Raza hispana. Hispanoamericanismo $e$ imaginario nacional en la España de la Restauración (Madrid, CEPC, 2010).

david.marcilhacy@sorbonne-universite.fr 